Proceedings

\title{
TGF- $\beta$ Inhibition Combined with Cytotoxic Nanomedicine Normalizes the Tumor Microenvironment and Improves Immune Checkpoint Inhibition Therapy ${ }^{\dagger}$
}

\author{
Myrofora Panagi ${ }^{1}$, Chrysovalantis Voutouri ${ }^{1}$, Fotios Mpekris ${ }^{1}$, John D. Martin ${ }^{2}$, Kazunori \\ Kataoka ${ }^{3,4}$, Horacio Cabral ${ }^{2}$ and Triantafyllos Stylianopoulos 1,* \\ 1 Cancer Biophysics Laboratory, Department of Mechanical and Manufacturing Engineering, University of \\ Cyprus, Nicosia 1678, Cyprus \\ 2 Department of Bioengineering, Graduate School of Engineering, The University of Tokyo, Bunkyo, Tokyo \\ 113-8654, Japan \\ 3 Innovation Center of NanoMedicine, Kawasaki Institute of Industrial Promotion, Kawasaki 210-0821, \\ Japan \\ 4 Policy Alternatives Research Institute, The University of Tokyo, Bunkyo, Tokyo 113-8654, Japan \\ * Correspondence: tstylian@ucy.ac.cy \\ + Presented at the 2nd International Online-Conference on Nanomaterials, 15-30 November 2020; Available \\ online: https://iocn2020.sciforum.net/.
}

Published: 15 November 2020

\begin{abstract}
Tumor normalization strategies aim to improve tumor blood vessel functionality (i.e., perfusion) by restoring tumor vessel compression and hyper-permeability. Despite progress in tumor normalization strategies, their combinatorial antitumor effects with nano- immunotherapy remain unexplored. In this presentation, we re-purposed the TGF- $\beta$ inhibitor tranilast, an approved anti-fibrotic and antihistamine drug, and combined it with Doxil nanomedicine to normalize murine models of triple negative breast cancer, increase perfusion and oxygenation, and enhance delivery and efficacy of Doxil and immune checkpoint blockers (ICBs). Specifically, we employed two triplenegative breast cancer mouse models to primarily evaluate the therapeutic and normalization effects of tranilast combined with Doxil. We demonstrated the optimized normalization effects of tranilast combined with Doxil and extended our analysis to investigate the effect of tumor normalization to the efficacy of ICBs. Combination of tranilast with Doxil caused a pronounced reduction in extracellular matrix components and increase in pericyte coverage, indicators of normalization. These modifications resulted in a significant increase in tumor perfusion and enhanced treatment efficacy as indicated by the notable reduction in tumor size. Furthermore, we found that combining tranilast with Doxil nanomedicine, significantly improved infiltration of $T$ cells into the tumor and the immunostimulatory M1 macrophage content and improved the efficacy of the anti-PD-1/anti-CTLA-4 treatment. We concluded that combinatorial treatment of tranilast with Doxil optimizes tumor normalization towards anti-tumor immunity.
\end{abstract}

Keywords: tumor microenvironment; normalization strategies; drug delivery; solid tumors

\section{Introduction}

The efficacy of nanomedicines to solid tumors is hindered by abnormalities in the structure of the tumor vasculature, which can reduce drastically tumor perfusion and as a result the systemic delivery of drugs, including chemotherapy, nanomedicine and immunotherapy [1-3]. A major abnormality in so called desmoplastic tumors (e.g., subtypes of breast, pancreatic and colon cancers and sarcomas) is the dense tumor extracellular matrix and the rapid proliferation of cancer cells in 
the confined space surrounding the tumor that stiffen the tumor and result in the accumulation of mechanical compressive forces that can cause compression of intratumoral blood vessels [4-8]. Vessel compression in turn results in reduced tumor blood flow, rendering tumors hypo-perfused and hypoxic. Impaired blood supply and hypoxia hinder nanoparticle delivery but also help cancer cells evade the immune system and increase their invasive and metastatic potential [9-11]. Particularly, hypo-perfusion can reduce the number of immune cells that can infiltrate into the tumor, while hypoxia renders the tumor microenvironment immunosuppressive and attenuates the killing potential of effector immune cells [11-14]. Normalization of the tumor interstitial space is a therapeutic strategy that has been proposed to normalize the tumor extracellular matrix in order to alleviate forces and decompress tumor vessels, thus, improving tumor perfusion. It is based on the use of drugs exerting anti-fibrotic properties and in previous studies we have re-purposed several approved drugs for this purpose [5,15-18]. The aim of the study was to investigate whether the normalization effects of tranilast, an approved in Japan and South Korea anti-fibrotic and antihistamine drug, can be optimized with use of subtherapeutic doses of Doxil. We demonstrate that combination of tranilast with Doxil nanomedicine, significantly improved blood vessel functionality and oxygenation and delayed tumor growth. Furthermore, the tranilast-Doxil treatment favored the accumulation of M1-like tumor associated macrophages (TAMs), presumably due to the increase in tumor oxygenation. Based on these data, we conducted a second study using the 4T1 tumor model to evaluate the therapeutic potential of immune checkpoint blockade combined with normalization of the tumor microenvironment. Again, we observed that the combinatorial tranilast-Doxil treatment significantly improved the efficacy of the immune checkpoint inhibition. Our findings strongly suggest that the effect of TGF- $\beta$ inhibition is drastically increased when combined with Doxil nanomedicine and can improve significantly immunotherapy.

\section{Materials and Methods}

Animal tumor models and treatment protocols. 4T1 and E0771 tumor models were generated by orthotopic implantation into the mammary fat pad of BALB/c and C57BL/6 female mice, respectively. Animals were treated with saline (Control), tranilast $(200 \mathrm{mg} / \mathrm{kg}$, orally), doxorubicin $(5 \mathrm{mg} / \mathrm{kg}$, intraperitoneal), Doxil (3 $\mathrm{mg} / \mathrm{kg}$, intravenously) or combination of tranilast-doxorubicin and tranilast-Doxil until time of death or time to reach a maximum tumor burden of $1200 \mathrm{~mm}^{3}$. Animal survival was monitored daily, while tumor size was measured every 2-3 days.

The effect of immunotherapy on tumor growth was evaluated in syngeneic 4T1 and E0771 tumor models. Immunotherapy was administrated as a cocktail of $10 \mathrm{mg} / \mathrm{kg}$ anti-PD-1 (CD279, clone RMP114, BioXCell, Lebanon, NH, USA) and $5 \mathrm{mg} / \mathrm{kg}$ anti-CTLA-4 (CD152, clone 9D9) following dilution in the recommended InVivoPure pH 7.0 Dilution Buffer (BioXCell). The immunotherapy cocktail was administered i.p. on days 14, 17 and 20 post-implantation. Animals were treated with saline, tranilast and Doxil as previously in combination with a non-targeting isotype control antibody (BioXCell).

Immunohistochemical assessment of tumor microenvironment. For fluorescent immunohistochemistry tumors were excised, fixed with $4 \%$ PFA and embedded in optimal temperature compound (OCT). Extracellular matrix content was studied using immunostaining against collagen I and hyaluronan. Pericyte coverage of functional vessels was determined as the ratio of lectin, $\alpha \mathrm{SMA}$ and $\mathrm{CD} 31$ positive staining to total CD31 area fraction.Macrophage status of 4T1 and E0771 TME was determined following immunostaining with anti-cd11c (HL3, BD Pharmingen 1:100), anti-CD206 (MR5D3, BIO-RAD 1:50) and rat anti-F4/80 (A3-1, BIO-RAD 1:50) antibodies to detect M1-like TAMs, M2-like TAMs and total TAM population, respectively. Quantification of M1-like TAMs was defined as the ratio of cd11c positive signal to $f 4 / 80$ area fraction. M2-like TAM content was indicated by cd206 area fraction after subtraction of the cd11c and cd206 overlapping signal. All measurements were normalized to DAPI positive staining. 


\section{Results}

\subsection{Doxil Enhances Tranilast-Induced Normalization of Tumor Extracellular Matrix}

Immunofluorescence staining of tumor sections shown that tranilast alone or in combination with doxorubicin or Doxil decreased collagen and hyaluronan content of both 4T1 and E0771 tumors, compared to control. When tranilast was administered with Doxil, collagen and hyaluronan levels were further significantly decreased (Figure 1A,C,D). Doxil enhances the interstitial space normalization effects of tranilast. Given the known anti-fibrotic properties of doxorubicin, this result can be attributed to the fact that tranilast enhances Doxil delivery and acts with Doxil to further reduce extracellular matrix components. Subsequently, the impact of the treatment on blood vessel pericyte coverage was studied, which is a measure of vascular normalization. In normal vessels, pericytes are attached to the vessel wall, whereas in tumors, pericyte coverage is poor thus contributing to increased vessel permeability. Immunofluorescence analysis of tumor sections counterstained with the CD31 endothelial marker and the $\alpha$ SMA marker depict the presence of pericytes attached to the tumor vasculature at the overlapping regions. Only animals treated with tranilast-Doxil exhibited a significant increase in pericyte coverage (Figure 1B,E), indicating vascular normalization.

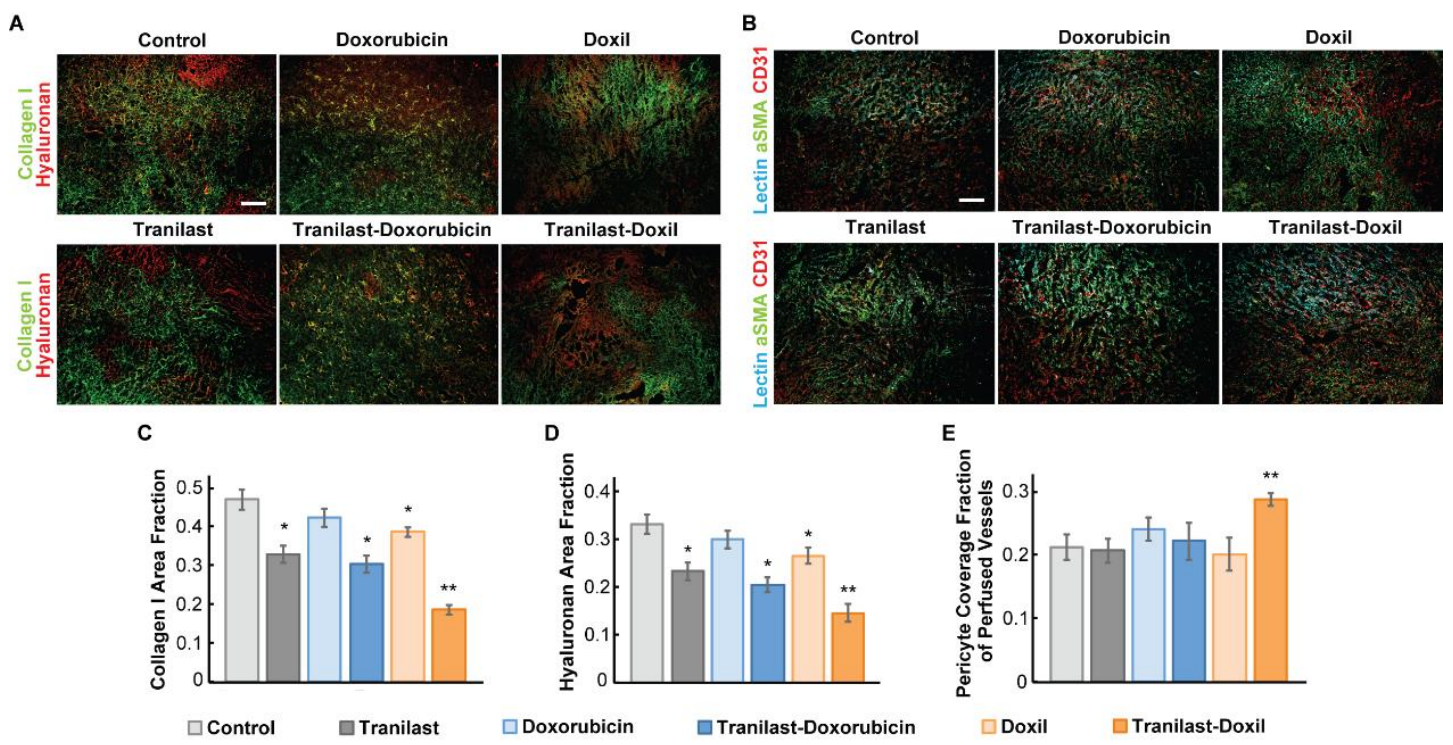

Figure 1. Doxil enhances tranilast-induced normalization. (A) Representative images of collagen (green) and hyaluronan (red) staining of 4T1 tumors. (B) Representative images of biotinylated tomato lectin (cyan), CD31 endothelial marker (red) and $\alpha$ SMA pericyte marker (green) immunostaining of $4 \mathrm{~T} 1$ breast tumors treated as indicated. Quantification of collagen (C), hyaluronan (D) area fractions and (E) pericyte coverage of perfused vessels. Statistical analyses were performed by comparing the treated groups with the control * and the tranilast-Doxil group with all other treatment groups ${ }^{* *}, p \leq 0.05,(n=8-10)$. Scale bar: $200 \mu \mathrm{m}$.

\subsection{Tranilast-Doxil Combinatorial Treatment Promotes M1 Macrophages Polarization}

Considering the normalization effects of tranilast-Doxil therapy on promoting interstitial and vascular normalization, which are related directly to improved perfusion and oxygenation, we performed immunofluorescence analysis of tumor sections stained with antibodies against CD11c and CD206 proteins, which are predominately expressed in M1- and M2-like tumor associated macrophages (TAMs), respectively. M2-TAMs are immunosuppressive and expressed under hypoxic conditions, whereas improved oxygenation can promote M1-phenotype and immunostimulation. Total macrophage population was calculated from the $f 4 / 80$ positive fraction (Figure 2A). Only the combination of tranilast with Doxil successfully increased the M1- to M2-like TAMs ratio, increasing the M1-like TAMs population (Figure 2B,C). Since the total amount of TAMs remained unaffected 
between the various treatment groups (Figure 2C), we concluded that tranilast-Doxil treatment redirects the M2-like immunosuppressive phenotype towards the M1-like immunosupportive TAMs.

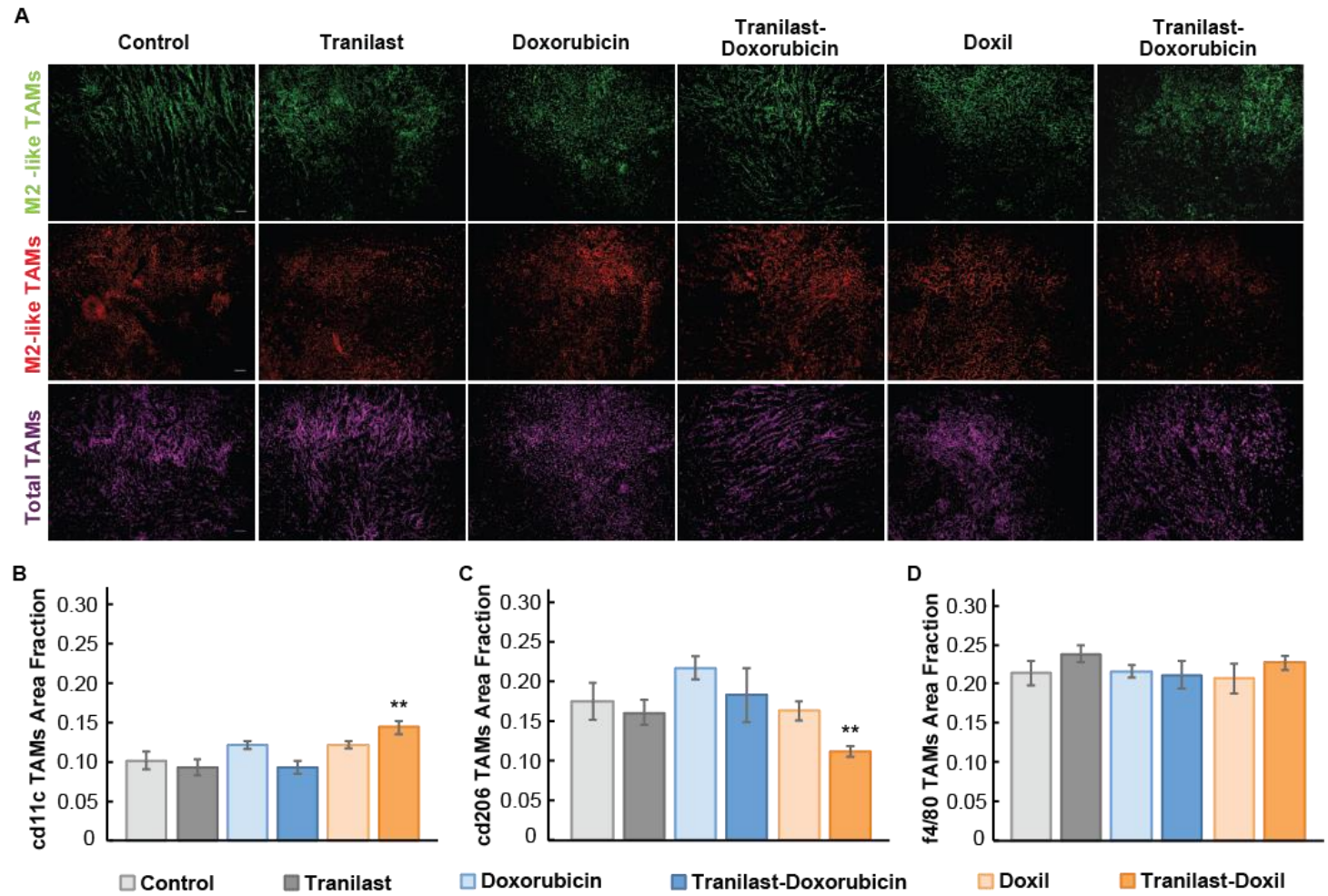

Figure 2. Tumor normalization in combination with Doxil but not doxorubicin reprograms macrophages by towards a M1 polarization. (A) Representative images of 4T1 tumors stained for the M1-like tumor associated macrophage (TAM) marker cd11c (green), the M2-TAM marker cd206 (red) and the $44 / 80$, which is a pan-macrophage marker (magenta). Quantification of anti-tumoral M1- to M2-like TAM ratios (B), pro-tumoral M2-like TAMs (C) and total TAMs area fraction (D) in the various treatment groups. Statistical analyses were performed by comparing the treated groups with the control ${ }^{*}$ and the tranilast-Doxil groups with all other treatment groups ${ }^{* *}, p \leq 0.05,(n=8-10)$. Scale bar: $200 \mu \mathrm{m}$.

\subsection{Tranilast Improves Efficacy of Nano-Immunotherapy}

To test our hypothesis that tumor normalization can improve the efficacy of nanoimmunotherapy, a tumor growth study was performed, employing a cocktail of immune checkpoint blockers (ICBs). Specifically, an immunotherapy cocktail was used consisting of the programmed cell death-1 (PD-1) and cytotoxic T-lymphocyte-associated antigen-4 (CTLA-4) antibodies. Interestingly, immunotherapy cocktail alone did not delay tumor growth, whereas Doxil treatment exhibited a modest decrease in tumor growth rate. Combination of immunotherapy with Doxil reduced tumor growth by $40 \%$ combared to the control, while its combination with the tranilast caused a reduction greater than $50 \%$ compared to the untreated group. Consistent with our previous results, tranilastDoxil combinatorial therapy significantly reduced tumor growth by $60 \%$. However, only the combination of tranilast with nano-immunotherapy treatment significantly reduced tumor volume in both mammary tumor models, 4T1 and E0771 (Figure 3). 
A

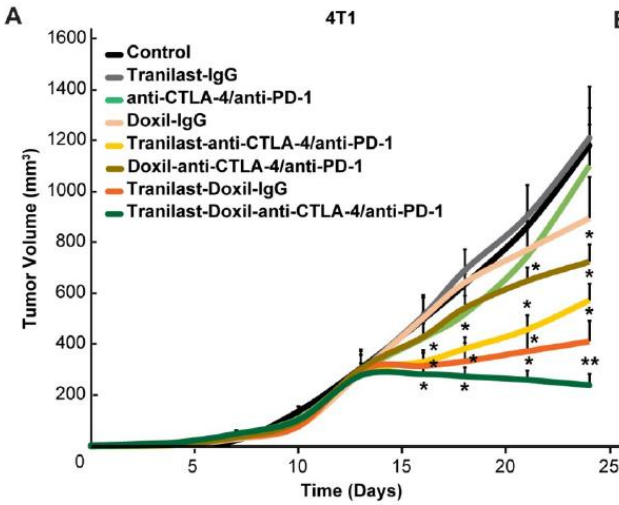

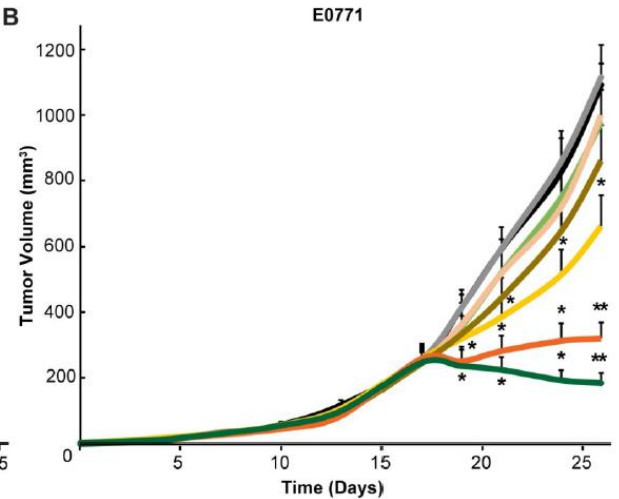

Figure 3. Tumor volume results of (A) $4 \mathrm{~T} 1$ and (B) E0771 tumors treated with saline (Control), tranilast (200 mg/kg), anti-CTLA4/anti-PD-1 immunotherapy cocktail $(5 \mathrm{mg} / \mathrm{kg}$ and $10 \mathrm{mg} / \mathrm{kg}$, respectively), Doxil (3 $\mathrm{mg} / \mathrm{kg})$, tranilast-immunotherapy, tranilast-Doxil, Doxil-immunotherapy and tranilast-Doxil-immunotherapy. Tranilast in combination with Doxil-immunotherapy inhibits tumor growth. Statistical analyses were performed by comparing the treated groups with the control * and the tranilast-Doxil-immunotherapy group with all other treatment groups ${ }^{* *}, p \leq 0.05(n=8-10)$.

\section{Discussion}

Our study suggests that tumor microenvironment normalization is enhanced when combined with cytotoxic nanomedicine. It also provides a rationale for combining tumor normalization agents nano- and immuno-therapies, given the enhanced therapeutic outcome upon the combination of the three. Importantly, a programmed death-ligand 1 (PD-L1) antibody (Tecentriq) combined with the nanomedicine (nab-paclitaxel chemotherapy Abraxane) has recently obtained FDA approval as the first immunotherapy drug for PD-L1 positive unresectable or metastatic triple negative breast cancers. Our findings also identify vessel compression as a main mechanism of resistance to nanoformulations of chemotherapy, which could explain to some extent why nanomedicines have not been successful in drastically increasing overall survival. It also provides new insights for the use of nanomedicine and the development of new nanoparticle formulations. We suggest that nanomedicine efficacy can be significantly improved in combination with agents that normalize the tumor microenvironment.

Author Contributions: Conceptualization, T.S.; methodology, all authors; formal analysis, all authors; writing original draft preparation, M.P.; writing-review and editing, all authors; supervision, T.S. All authors have read and agreed to the published version of the manuscript.

Funding: This work was supported by the European Research Council Grant ERC-2013-StG-336839 and the INFRASTRUCTURE/1216/0052 grant co-financed by the European Regional Development Fund and the Republic of Cyprus through the Research Promotion Foundationthe Research Promotion Foundation of Cyprus (INFRASTRACTURES/1216/0052) (to T.S.) and partially supported by Grants-in-Aid for Scientific Research B (JP16H03179; H.C.). JDM was supported by a fellowship from the Japan Society for the Promotion of Science (No. P16731).

Acknowledgments: A complete version of this work has been published in Panagi, M.C. Voutouri, F. Mpekris, P. Papageorgis, M.R. Martin, J.D. Martin, P. Demetriou, C. Pierides, C. Polydorou, A. Stylianou, M. Louca, M., L. Koumas, P. Costeas, K. Kataoka, H. Cabral and T. Stylianopoulos. TGF- $\beta$ inhibition combined with cytotoxic nanomedicine normalizes triple negative breast cancer microenvironment towards anti-tumor immunity. Theranostics, (4):1910-1922. [DOI:10.7150/thno.36936, https://www.thno.org/v10p1910.htm].

Conflicts of Interest: The authors declare no conflict of interest.

\section{References}

1. Jain, R.K.; Stylianopoulos, T. Delivering nanomedicine to solid tumors. Nat. Rev. Clin. Oncol. 2010, 7, 653664, doi:10.1038/nrclinonc.2010.139. 
2. Martin, J.D.; Cabral, H.; Stylianopoulos, T.; Jain, R.K. Improving cancer immunotherapy using nanomedicines: Progress, opportunities and challenges. Nat. Rev. Clin. Oncol. 2020, 17, 251-266.

3. Stylianopoulos, T.; Munn, L.L.; Jain, R.K. Reengineering the Physical Microenvironment of Tumors to Improve Drug Delivery and Efficacy: From Mathematical Modeling to Bench to Bedside. Trends Cancer 2018, 4, 292-319, doi:10.1016/j.trecan.2018.02.005.

4. Stylianopoulos, T.; Martin, J.D.; Snuderl, M.; Mpekris, F.; Jain, S.R.; Jain, R.K. Coevolution of solid stress and interstitial fluid pressure in tumors during progression: Implications for vascular collapse. Cancer Res. 2013, 73, 3833-3841, doi:10.1158/0008-5472.can-12-4521.

5. Stylianopoulos, T.; Martin, J.D.; Chauhan, V.P.; Jain, S.R.; Diop-Frimpong, B.; Bardeesy, N.; Smith, B.L.; Ferrone, C.R.; Hornicek, F.J.; Boucher, Y.; et al. Causes, consequences, and remedies for growth-induced solid stress in murine and human tumors. Proc. Natl. Acad. Sci. USA 2012, 109, 15101-15108, doi:10.1073/pnas.1213353109.

6. Voutouri, C.; Stylianopoulos, T. Accumulation of mechanical forces in tumors is related to hyaluronan content and tissue stiffness. PLoS ONE 2018, 13, e0193801, doi:10.1371/journal.pone.0193801.

7. Voutouri, C.; Polydorou, C.; Papageorgis, P.; Gkretsi, V.; Stylianopoulos, T. Hyaluronan-Derived Swelling of Solid Tumors, the Contribution of Collagen and Cancer Cells, and Implications for Cancer Therapy. Neoplasia 2016, 18, 732-741, doi:10.1016/j.neo.2016.10.001.

8. Jain, R.K.; Martin, J.D.; Stylianopoulos, T. The role of mechanical forces in tumor growth and therapy. Annu. Rev. Biomed. Eng. 2014, 16, 321-346.

9. Facciabene, A.; Peng, X.; Hagemann, I.S.; Balint, K.; Barchetti, A.; Wang, L.P.; Gimotty, P.A.; Gilks, C.B.; Lal, P.; Zhang, L.; et al. Tumour hypoxia promotes tolerance and angiogenesis via CCL28 and T(reg) cells. Nature 2011, 475, 226-230, doi:10.1038/nature10169.

10. Jain, R.K. Antiangiogenesis strategies revisited: From starving tumors to alleviating hypoxia. Cancer Cell 2014, 26, 605-622, doi:10.1016/j.ccell.2014.10.006.

11. Semenza, G.L. Oxygen sensing, hypoxia-inducible factors, and disease pathophysiology. Annu. Rev. Pathol. 2014, 9, 47-71, doi:10.1146/annurev-pathol-012513-104720.

12. Wilson, W.R.; Hay, M.P. Targeting hypoxia in cancer therapy. Nat. Rev. Cancer 2011, 11, 393-410, doi:10.1038/nrc3064.

13. Motz, G.T.; Coukos, G. Deciphering and reversing tumor immune suppression. Immunity 2013, 39, 61-73, doi:10.1016/j.immuni.2013.07.005.

14. Palazon, A.; Aragones, J.; Morales-Kastresana, A.; de Landazuri, M.O.; Melero, I. Molecular pathways: Hypoxia response in immune cells fighting or promoting cancer. Clin. Cancer Res. 2012, 18, 1207-1213, doi:10.1158/1078-0432.CCR-11-1591.

15. Chauhan, V.P.; Martin, J.D.; Liu, H.; Lacorre, D.A.; Jain, S.R.; Kozin, S.V.; Stylianopoulos, T.; Mousa, A.; Han, X.; Adstamongkonkul, P.; et al. Angiotensin inhibition enhances drug delivery and potentiates chemotherapy by decompressing tumor blood vessels. Nat. Commun. 2013, 4, 1-11, doi:10.1038/ncomms.3516.

16. Polydorou, C.; Mpekris, F.; Papageorgis, P.; Voutouri, C.; Stylianopoulos, T. Pirfenidone normalizes the tumor microenvironment to improve chemotherapy. Oncotarget 2017, 8, 24506-24517, doi:10.18632/oncotarget.15534.

17. Mpekris, F.; Papageorgis, P.; Polydorou, C.; Voutouri, C.; Kalli, M.; Pirentis, A.P.; Stylianopoulos, T. Sonichedgehog pathway inhibition normalizes desmoplastic tumor microenvironment to improve chemo- and nanotherapy. J. Control. Release 2017, 261, 105-112, doi:10.1016/j.jconrel.2017.06.022.

18. Zhao, Y.; Cao, J.; Melamed, A.; Worley, M.; Gockley, A.; Jones, D.; Nia, H.T.; Zhang, Y.; Stylianopoulos, T.; Kumar, A.S.; et al. Losartan treatment enhances chemotherapy efficacy and reduces ascites in ovarian cancer models by normalizing the tumor stroma. Proc. Natl. Acad. Sci. USA 2019, 116, 2210-2219, doi:10.1073/pnas.1818357116.

Publisher's Note: MDPI stays neutral with regard to jurisdictional claims in published maps and institutional affiliations.

(C) 2020 by the authors. Submitted for possible open access publication under the terms and conditions of the Creative Commons Attribution (CC BY) license (http://creativecommons.org/licenses/by/4.0/). 